\title{
Médiévales
}

Langues, Textes, Histoire

54 | printemps 2008

Frères et sœurs

\section{Seules ou accompagnées? Les veuves parisiennes et leurs fratries à la fin du Moyen Âge}

Parisian widows and their sibship.

Caroline Jeanne

\section{(2) OpenEdition}

1 Journals

Édition électronique

URL : https://journals.openedition.org/medievales/4872

DOI : $10.4000 /$ medievales.4872

ISSN : $1777-5892$

Éditeur

Presses universitaires de Vincennes

Édition imprimée

Date de publication : 1 juin 2008

Pagination : 69-81

ISBN : 978-2-84292-217-7

ISSN : 0751-2708

\section{Référence électronique}

Caroline Jeanne, «Seules ou accompagnées? Les veuves parisiennes et leurs fratries à la fin du Moyen Âge », Médiévales [En ligne], 54 I printemps 2008, mis en ligne le 10 septembre 2010, consulté le 22 avril 2022. URL : http://journals.openedition.org/medievales/4872 ; DOI : https://doi.org/ 10.4000 /medievales.4872

Ce document a été généré automatiquement le 22 avril 2022.

Tous droits réservés 


\title{
Seules ou accompagnées? Les veuves parisiennes et leurs fratries à la fin du Moyen Âge
}

Parisian widows and their sibship.

\author{
Caroline Jeanne
}

1 Le mot « veuve » définit en premier lieu l'identité d'une femme en référence à son mari défunt ${ }^{1}$. Il inscrit d'emblée l'identité d'une femme dans un lien conjugal qui s'est défait. Or ce lien conjugal, aux XIV et XV siècles, implique bien davantage de personnes qu'un seul couple, un homme et une femme unis par les liens du mariage. Il attache chacun des conjoints à deux groupes familiaux, celui de l'époux et celui de l'épouse. À la mort de l'époux, se pose ainsi la question de la position des veuves par rapport à ces deux groupes : quelle place, quel rôle et quel statut sont assignés aux veuves au sein du groupe familial dont elles sont issues, mais aussi au sein du groupe familial auquel elles appartiennent par alliance?

2 Le questionnement sur le lien de fraternité permet en partie d'apporter des éléments de réponse. Se demander si la rupture du lien matrimonial influe sur la relation qui unit les veuves à leurs fratries permet de mesurer la force et les redéfinitions des attaches familiales dans le contexte particulier du veuvage, moment où les liens familiaux sont à bien des égards bouleversés. Définies par un statut spécifique qui diffère nettement de celui des femmes mariées, les veuves doivent en effet endosser et affirmer un rôle nouveau auprès de leurs frères et sœurs. Ce statut, du point de vue juridique, apparaît plus proche de celui de la femme séparée que de celui de la femme mariée. Il se caractérise, dans le Paris de la fin du Moyen Âge, par le recouvrement d'une capacité qui autorise les veuves à agir et être assignées en justice sans autorisation maritale et, théoriquement, à recouvrer la maitrise de leurs biens².

3 Compte tenu de cet ancrage des veuves dans deux groupes familiaux, la relation de fraternité doit ici être comprise au sens large du terme : elle envisage d'une part les liens des veuves avec leurs frères et sœurs germains, mais aussi avec les conjoints de ceux-ci. Elle inclut d'autre part les relations que les veuves entretiennent avec les 
frères et sœurs de leur mari défunt, et avec les conjoints de ceux-ci. Frères, sœurs, beaux-frères et belles-sœurs forment ainsi autour des veuves un véritable entourage fraternel, au sein duquel se nouent des relations particulières ${ }^{3}$, alors même que le statut des veuves demeure fortement associé à l'idée de privation, d'abandon et de solitude ${ }^{4}$.

Dans les sources parisiennes de la fin du Moyen Âge, qui nous renseignent sur un milieu aisé, les relations des veuves avec leurs frères et sœurs sont inégalement perceptibles. Certains types de documents les éludent complètement. Ainsi, dans les sources fiscales $\mathrm{du}$ quinzième siècle, une analyse anthroponymique montre que les modes de désignation des veuves font intervenir presque systématiquement un référent masculin. Il s'agit toujours du mari défunt ou du père, mais jamais du frère ou du beaufrère: les veuves sont avant tout "femme de feu " ou "fille de", mais ne sont pas considérées comme «sœur de... » ${ }^{5}$. De même, aucun feu fiscal féminin dirigé par une veuve ne fait mention d'un frère ou d'une sœur ${ }^{6}$. Cependant, ces rôles d'impôts ne sont pas représentatifs de l'ensemble des sources qui sont à notre disposition : si les veuves sont généralement identifiées au sein de la population parisienne par le rappel du nom de leur père ou de leur mari défunt, ceci n'exclut pas une relation forte aux frères et sœurs, d'autant plus importante qu'elle s'avère bien souvent plus durable que celle qui unit les veuves à leur père ou à leur mari. Si nous nous tournons par exemple vers les testaments, les actes notariés ${ }^{7}$ ou la documentation judiciaire de la fin du Moyen Âge ${ }^{8}$, ces relations de fraternité apparaissent beaucoup plus distinctement et permettent d'entrevoir la richesse et la complexité des liens qui unissent les veuves à leurs fratries. Dans le cadre de relations qui vont de l'entraide fraternelle aux conflits menés jusque devant les tribunaux, les portraits de veuves sont divers et nuancés, à l'image du statut de ces femmes. Fragiles ou autonomes, miserabiles personae ou femmes viriles, les veuves parisiennes apparaissent vis-à-vis de leurs frères et sœurs tour à tour comme les protégées, des interlocutrices à part entière ou les plus redoutables des adversaires.

Des sœurs aux multiples visages : les veuves protégées par leurs frères

5 Le devoir d'assistance envers les veuves est un thème récurrent au Moyen Âge. Les veuves sont traditionnellement présentées comme des personnes devant naturellement bénéficier de soutien, d'aide et de charité. C'est là une mission royale et un lieu commun dans la théorie politique médiévale, comme le montrent bien les formules des ordonnances royales ${ }^{9}$. C'est également une mission de l'Église: le droit canonique établit l'appartenance des veuves à la catégorie des miserabiles personae, dont l'Église doit prioritairement assurer la protection ${ }^{10}$.

6 L'examen des relations des veuves avec leurs frères et sœurs semble montrer que la fratrie s'attribue de la même façon une mission d'assistance envers ses veuves. Nous sommes ici dans le domaine des solidarités familiales, dont les veuves apparaissent nettement comme des bénéficiaires privilégiées et prioritaires. Les legs testamentaires en fournissent de nombreux exemples. Ainsi, en 1407, le codicille qu'Arnaud de Corbie rédige à la suite de la mort de son frère Thomas contient une disposition particulière pour sa belle-sœur devenue veuve, qu'il désigne comme "ma suer, damoiselle Marguerite de Cresecques, femme de feu Thomas de Corbie, mon frere, ou temps qu'il vivoit $^{11} »$. L'expression du lien adelphique se passe ici de la distinction entre sœur germaine et sœur par alliance: la veuve du frère est ici pleinement sœur, sans restriction d'aucune sorte. Bien plus, le codicille tient compte du nouveau statut de veuve de Marguerite en prévoyant pour elle, outre le legs déjà mentionné dans le 
testament initial, des dispositions veillant à confirmer et amplifier celles de Thomas pour sa femme, afin que pendant son veuvage, Marguerite bénéficie de six cents livres parisis de rente. On voit ici s'exprimer une attention toute particulière envers une belle-sœur devenue veuve, et qui apparaît de ce fait plus vulnérable. Le devoir de protection du mari envers sa femme et future veuve est ici complètement relayé par le frère du défunt: le beau-frère se doit, lui aussi, de veiller au mieux au devenir de Marguerite, et au maintien de son statut social. Cette attention portée au lien adelphique dans les testaments se retrouve dans celui qu'établit Renaud de Trie en 1406 : lorsqu'il prend des dispositions pour sa femme et future veuve, il désigne celle-ci comme «ma femme, compaigne et amie, principale et premiere, ma sereur ${ }^{12} » . \mathrm{Ce}$ terme de référence qui compare l'épouse à une sœur fait du lien matrimonial de qualité l'équivalent du lien adelphique : dans son testament, le mari veut protéger sa future veuve comme il protègerait une sœur, c'est-à-dire de façon privilégiée. La valorisation $\mathrm{du}$ lien adelphique dans le souci du devenir des veuves est récurrente.

7 La protection des veuves par leur fratrie apparaît ailleurs et selon d'autres modalités que dans les legs testamentaires. Les archives notariées, rendant compte de divers aspects de la vie économique ou privée des Parisiens $d u \mathrm{xv}^{\mathrm{e}}$ siècle, apportent des éléments complémentaires et concordants. Ainsi, les exemples de procurations ayant pour objet la représentation en justice ou l'administration de biens associent très souvent une veuve à son frère procureur. D'autres exemples montrent pourtant que les veuves peuvent ester. En 1492, Martine, veuve d'un laboureur parisien, donne procuration à son frère Jean Montoysel pour qu'il la représente en justice et gère ses bien $^{13}$. En 1497, c'est également à son frère que Jeanne, veuve de Jean Varlier, marchand et bourgeois de Paris, donne procuration pour l'administration de ses biens ${ }^{14}$. Ces deux exemples parmi d'autres illustrent le besoin d'assistance de veuves peinant à faire valoir leurs droits. En ce qui concerne la représentation en justice, ces procurations font écho aux écrits de Christine de Pizan déplorant les difficultés pour une veuve de défendre seule ses droits devant les tribunaux ${ }^{15}$ : elle-même en a fait l'expérience après la mort de son mari, en 1390, quand elle dut défendre ses intérêts dans plusieurs affaires relatives aux biens de son mari ou aux rentes dont il était chargé ${ }^{16}$. Quant à la gestion des biens, il s'agit là d'un rôle traditionnellement accaparé par le mari ${ }^{17}$, que les veuves peuvent s'approprier du fait de leur changement de statut, mais qui peut aussi leur être contesté, ou en tout cas être entravé. Dans ces deux situations, qui donnent lieu à des procurations, les frères apparaissent comme les successeurs des pères, et surtout des maris, auprès de veuves qui en sont désormais dépourvues. Le lien fraternel se substitue ainsi au lien marital.

8 Ainsi, dans le cadre des legs testamentaires ou des actes notariés qui instaurent d'une manière ou d'une autre un système de protection des veuves par leur fratrie, la référence au masculin domine largement: dès lors qu'il s'agit de l'assistance des veuves, et notamment d'une assistance matérielle, sœurs et belles-sœurs sont le plus souvent absentes. La protection est avant tout le fait des frères et des beaux-frères, agissant en ceci envers les veuves comme les remplaçants privilégiés des maris défunts ${ }^{18}$. C'est là un comportement attendu de la part des membres masculins de la fratrie. Cependant il arrive aux veuves de déjouer ces assignations identitaires genrées, lorsqu'elles œuvrent pour prêter service ou assistance à leurs frères. La solidarité fraternelle s'exerce alors dans l'autre sens: ce sont des veuves qui la dispensent, agissant ainsi peut-être comme des mères de substitution ${ }^{19}$. À la fin du $\mathrm{Xv}^{\mathrm{e}}$ siècle, deux actes notariés montrent que Justine Sanguin, veuve d'un conseiller au Parlement, cède 
à bail ou prête à ses frères Nicaise et Antoine des maisons à Paris, rue du Roi-de-Sicile ${ }^{20}$. Les actes sont muets quant à une éventuelle contrepartie, et il semble bien qu'il ne soit pas question ici d'une protection fraternelle envers la veuve, mais de la situation inverse. Il serait dès lors intéressant, si les sources étaient moins laconiques, d'éclairer ces cas en prenant en compte l'âge, et l'éventuel rôle d'aînée joué par la veuve, ou le niveau de fortune, parfois plus décisifs que le rapport de genre. L'influence de l'âge explique peut-être le transfert de créances opéré par Marguerite Philippon, veuve d'un notaire et secrétaire du roi, envers son frère écolier à l'université de Paris, pour permettre à celui-ci d'aller devant la justice ecclésiastique ${ }^{21}$ : la solidarité fraternelle joue pleinement, au bénéfice d'un frère profitant de la protection complice de sa sœur veuve. Enfin, les veuves n'oublient pas leur fratrie dans leurs legs testamentaires : en 1420, Simonnette la Maugère, veuve de Robert Mauger, laisse à sa sœur Jeanne « cent escuz en or et [son] manteau fourré de gris » et à sa nièce, fille de sa sœur Marguerite, des vêtements et une somme d'argent pour son mariage ${ }^{22}$.

Si les veuves apparaissent comme les premières bénéficiaires d'une protection fraternelle essentiellement masculine, des exemples montrent qu'elles sont parfois en mesure elles aussi de fournir une assistance, de façon personnelle. Ainsi les veuves sont-elles au cœur de l'entraide fraternelle, dont elles profitent de façon prioritaire et qu'elles prodiguent quand elles le peuvent.

Interlocutrices à part entière dans la fratrie

Le rôle des veuves, loin de se cantonner à celui de miserabiles personae nécessitant une protection, révèle d'autres facettes, et les documents de la pratique se montrent plus diserts sur ce point que les écrits des théologiens. Ainsi, au sein de la fratrie, on retrouve les veuves impliquées dans divers types d'affaires ou de transactions aux côtés de leurs frères et sœurs. L'absence de mari aurait pu conduire à l'hypothèse de leur éviction des tractations ou prises de décision concernant la fratrie. Il n'en est rien : on trouve trace de veuves, en nombre, dans des actes notariés de nature très variée, aux côtés de leurs frères et sœurs. Elles se comportent alors en interlocutrices à part entière. Ceci découle en premier lieu du pouvoir de chef de famille qui leur est imparti du fait de leur viduité. Il faudrait, pour mesurer finement ce pouvoir, prendre en compte dans l'analyse le critère décisif de l'âge des veuves, ou de leur position dans le cycle de vie : les sources ne le permettent pas toujours.

11 Dans les affaires concernant leur famille d'origine, succession des parents ou d'un membre de la fratrie par exemple, les veuves semblent tenir leur place au même titre que leurs frères et sœurs. Elles paraissent même jouir d'un statut plus autonome et affirmé que leurs sœurs mariées, puisque ces dernières sont toujours associées à leur époux qui, en tant que beau-frère, n'est jamais en retrait. Ainsi, en 1499, Marie Turquam, veuve d'un procureur au Châtelet, conclut avec ses deux frères Philippe et Robert, sa sœur Marguerite et l'époux de celle-ci un accord pour achever un procès au sujet de la succession de leur frère Jean ${ }^{23}$. Les quatre frères et sœurs s'entendent pour clore les discussions et la veuve, parmi eux, a voix au chapitre comme les autres. De même, quand les enfants de Marion, veuve d'Étienne Guespin, lui constituent une rente pour paiement de son douaire coutumier, sa fille Chrétienne, veuve elle aussi, a tout crédit pour prendre part à la décision et apparaît au même titre que les autres enfants dans l'acte notarié ${ }^{24}$.

Si les veuves sont plus souvent associées à leurs frères qu'à leurs sœurs en affaires, pour des ventes, baux et autres tractations de nature économique ou privée, elles se 
rendent parfois aussi devant le notaire avec une sœur. Les relations sororales ne sont pas absentes de ces documents. En 1498, deux sœurs veuves, exécutrices testamentaires de leur demi-sœur, délivrent une rente à la fabrique de l'église Saint-Paul ${ }^{25}$. Un acte de 1496 met plus nettement encore en évidence une complicité unissant deux sœurs veuves : il s'agit d'un acte de notoriété par lequel l'une d'entre-elle, Jeanne Lablonde, témoigne des maltraitances conjugales subies par sa nièce, fille de sa sœur Lucette ${ }^{26}$. L'acte précise que Jeanne atteste des faits à la requête de sa sœur Lucette. Dans chacun de ces cas, et surtout dans le second, le lien de sororité est peut-être renforcé par le fait que les deux sœurs partagent le même statut de veuve, qui les autorise et les incite à davantage d'autonomie et d'affirmation. Ces veuves ne sont pas accompagnées ou représentées par des hommes. Quoi qu'il en soit, ces exemples montrent bien que le statut des veuves est pluriel: ces femmes tiennent certes bien souvent le rôle de « remplaçante » de leur mari défunt dans diverses affaires, en tant que nouveau chef de famille, mais elles jouent aussi pleinement leur rôle de sœur.

13 Par ailleurs, il convient de nuancer en fonction de la fratrie avec laquelle les veuves sont en affaires: leur fratrie germaine ou la fratrie de leur mari défunt. Le cas d'Isabeau, veuve de Jean Gaultier, renseigné par trois actes notariés, en offre une illustration. Par un acte de 1500, cette veuve établit un bail, portant sur des biens immeubles hérités de sa mère, à Pierre Guillier, mari de sa sœur ${ }^{27}$. Il faut noter qu'Isabeau traite ici avec son beau-frère et non avec sa sœur qui, en tant que femme mariée, s'efface derrière son mari pour tout ce qui concerne la gestion des biens. Le masculin l'emporte sur la relation adelphique, en même temps que la mort du mari permet à la veuve d'entrer dans une relation de relative égalité avec un autre homme de la parentée ${ }^{28}$. À la même date, Isabeau règle une dette que son mari avait contractée de son vivant envers Pierre Guillier: elle solde ainsi les comptes de son mari, tâche incombant traditionnellement aux veuves ${ }^{29}$. Deux actes nous font apercevoir les relations qu'elle entretient avec un autre beau-frère, Jacques Gaultier, frère de son mari défunt, et tuteur des enfants d'Isabeau ${ }^{30}$. Avec ce dernier, elle semble plutôt nouer des liens d'association, prolongeant sans doute les affaires que menaient en commun les deux frères, et agissant comme représentante des intérêts des enfants : la veuve et son beau-frère sont associés dans un bail en 1495, et dans une vente en 1498. Dès lors, c'est une véritable complémentarité des rôles qui semble se mettre en place pour pallier l'absence du défunt en tant que frère et en tant que père : la veuve remplace son mari défunt dans son rôle de frère pour les affaires, le beau-frère remplace le père auprès des enfants ${ }^{31}$. Au sein de la fratrie de son époux défunt, la veuve tient ici une place de sœur à part entière et apparaît comme une interlocutrice privilégiée.

La nomination de tuteurs et curateurs offre un autre éclairage sur les liens qu'entretiennent les veuves avec leurs frères et sœurs dans le cadre d'affaires familiales, liens qui découlent dans ce cas directement du veuvage. Le registre des causes civiles de l'officialité épiscopale de Paris, de la fin du XIv e siècle, permet de préciser cet aspect ${ }^{32}:$ il contient en effet un certain nombre d'affaires concernant les dations de tuteurs et de curateurs, intervenant au cours d'un intervalle plus ou moins long après le décès d'un des parents, du père dans les cas qui nous intéressent ici. La procédure de dation s'effectuait en deux temps ${ }^{33}$. Dans un premier temps, les tuteurs et curateurs sont présentés par une sorte de conseil de famille, assemblée de parents dont les veuves font très souvent partie ${ }^{34}$. Dans un second temps, l'official ratifie le choix des tuteurs et curateurs et procède à la dation proprement dite. L'analyse du registre montre que là encore les sœurs ou belles-sœurs des veuves ne sont jamais mentionnées. 
En revanche, les oncles (avunculus, qui désigne indifféremment à cette époque l'oncle maternel ou paternel, plus souvent que patruus) sont très présents dans ce type d'affaires, participant à l'assemblée de parents aux côtés des veuves, ou désignés comme tuteurs et curateurs des enfants. On retrouve, avec cette importance du lien avunculaire, un trait caractéristique et bien repéré des structures de parenté médiévales ${ }^{35}$. Les frères et beaux-frères des veuves jouent donc un rôle non négligeable à leurs côtés en ce qui concerne les enfants : c'est là une autre manifestation des liens qui unissent les veuves à leur fratrie. En exerçant la tutelle et la curatelle de leurs neveux et nièces, les frères et beaux-frères sont nécessairement en contact étroit avec les veuves, contact dont la nature peut varier du conseil bienveillant à l'exercice d'une autorité plus ou moins contraignante.

Des veuves adversaires de leurs frères et soeurs

Beaucoup d'affaires dans lesquelles sont impliqués les veuves et leurs frères et sœurs (successions, tutelle des enfants) se règlent à l'amiable, et certaines d'entre elles relèvent même, nous l'avons vu, de l'association (ventes, baux). Ces cas existent en nombre, et illustrent les relations ordinaires nouées entre les veuves et leur fratrie. Cependant, les veuves parisiennes se rendent également devant les tribunaux dans le cadre de situations bien plus conflictuelles. Les cas de frères et sœurs se déchirant en justice existent eux aussi, et, dans le cas des veuves, ils apparaissent tout particulièrement au sujet de la succession du mari et frère défunt. Très souvent en effet, le décès ouvre une période de crise au cours de laquelle le partage du patrimoine laissé sans titulaire doit être effectué. Cette période de deuil est aussi celle pendant laquelle les veuves sont susceptibles d'apparaître comme les adversaires les plus redoutables des frères et sœurs du défunt, dans les configurations où ces derniers sont les héritiers les plus proches. Les cas que nous examinerons ici montrent donc, s'il en était besoin, que les relations que les veuves entretiennent avec leur belle-famille sont parfois houleuses. Ils viennent ainsi nuancer fortement l'image du beau-frère protecteur envers la veuve de son frère précédemment décrite.

16 Le registre des causes civiles du Châtelet de Paris recèle de tels exemples de conflits. En 1505 , une affaire relative à la succession de Christophe des Vignes oppose la veuve de ce dernier, Marie Defreux, et la sœur du défunt, Anne des Vignes ${ }^{36}$. Les deux femmes se déchirent au sujet des "partages et divisions" devant être faits des "conquestz et biens immeubles demourez du decès dudit deffunct ». Le couple ne semble pas avoir eu d'enfant, et Anne des Vignes, en tant que sœur du défunt, est l'héritière la plus proche. Il s'agit donc clairement d'un conflit opposant deux positions, celle de la veuve et celle de l'héritière collatérale. Sans entrer dans les détails de l'affaire, il apparaît que Marie Defreux se montre réticente à " desplacer et delivrer » les biens issus de la succession, dont une partie doit revenir à sa belle-sœur. Ces difficultés pour concilier les saisines mènent les deux protagonistes devant le Châtelet. Elles s'expliquent sans doute par des rivalités assez courantes entre héritiers, qui opposent dans ce cas deux femmes, mais aussi peut-être par le problème sous-jacent de la délivrance de leur douaire aux veuves. En effet, la mise en possession du douaire, ce gain de survie accordé à la femme survivante sur les biens propres de son mari défunt ${ }^{37}$, est souvent problématique et source de divergences avec les héritiers du mari qui en entravent la délivrance. Dans cette affaire, la veuve et sa belle-sœur sont en rivalité, l'une parce qu'elle fut l'épouse du défunt, l'autre parce qu'elle fut sa sœur : notons cependant que devant le Châtelet, elles ne comparaissent pas seules mais chacune avec son mari, la veuve étant remariée. Le cercle des protagonistes de l'affaire s'élargit donc aux conjoints : si Marie Defreux 
agit en tant que veuve, ce n'est pas pour autant qu'elle se rend seule devant le tribunal, puisqu'elle est remariée ${ }^{38}$.

Une autre affaire, similaire sur le fond, oppose en 1399 la veuve de Regnaut de la Mote, Jehanne la Coquatrisse, et le frère de celui-ci, Guillaume de la Mote. Les deux parties sont en " debat et question, pour raison des biens demourés du decès dudit deffunct ${ }^{39}$. " L'héritier et la veuve peinent à concilier leurs prétentions sur la succession du défunt, et Guillaume accuse sa belle-sœur d'avoir indûment "troubl[é] et empesch[é] ses possessions et saisines ${ }^{40}$.» Plus précisément, la veuve s'oppose à son beau-frère sur deux fronts distincts. D'une part, en tant que veuve, elle se montre vigilante en ce qui concerne la délivrance d'un don mutuel conclu du vivant de son mari. En effet, le conjoint survivant n'était pas saisi du don mutuel : il devait s'adresser, pour en obtenir la remise, aux héritiers du défunt, d'où nombre d'actions en délivrance dirigées contre les héritiers. D'autre part, en tant qu'exécutrice testamentaire de son mari ${ }^{41}$, la veuve se heurte aux prétentions de son beau-frère, héritier : à partir de la fin du XIV siècle, en effet, les héritiers réclament et se font progressivement reconnaître le droit d'accomplir le testament, à condition de fournir des sûretés garantissant l'exécution fidèle des dernières volontés du défunt. Ils entendent ainsi se substituer aux exécuteurs testamentaires, dont ils mettent en péril la saisine ${ }^{42}$. Dans cette affaire, le Châtelet donne la récréance aux exécuteurs : la veuve se voit donner raison, mais son beau-frère en appelle en Parlement. Ainsi, l'antagonisme qui tend les relations entre Jehanne la Coquatrisse et Guillaume de la Mote est certes un conflit entre une veuve et son beaufrère; mais plus précisément, analysé en termes juridiques, il relève d'une double opposition d'intérêts: entre l'héritier et la conjointe du défunt d'une part, entre l'héritier et l'exécutrice testamentaire d'autre part. Au-delà du terme général de veuve ou de belle-soeur, il convient donc de bien repérer les différents rôles et statuts des femmes qui ont perdu leur mari et s'affrontent aux frères et sœurs de celui-ci devant les tribunaux.

18 Les problèmes successoraux ne sont pas les seuls à l'origine de heurts entre les veuves et leurs frères et sœurs. Le remariage constitue par exemple une autre pierre d'achoppement, comme le suggère une affaire portée devant l'officialité de Paris: Agnès, veuve de Simon de Maubeuge, a été fiancée par son frère, maître en théologie, mais il apparaît que la ratification de la fiancée n'a pas été obtenue, ce qui remet en cause la validité des fiançailles ${ }^{43}$. Le procès a pour objet de vérifier si cette ratification, indispensable, a bien été consentie par la veuve. Or, celle-ci semble ici avoir été fiancée d'autorité par son frère. L'exercice d'une tutelle fraternelle contraignante sur les veuves est un phénomène récurrent dans les affaires de remariage. Ce contrôle des frères montre à quel point le remariage des veuves remet en cause l'autonomie de ces dernières, parce que des intérêts familiaux sont en jeu ${ }^{44}$. Il semble ainsi que le veuvage soit parfois l'occasion de réactiver l'autorité de la famille d'origine de la veuve sur celle-ci, autorité qui s'était effacée lors du mariage. Les frères prennent ainsi le relais des pères pour maintenir cette surveillance masculine sur les femmes de la famille qui en sont désormais dépourvues.

19 Les relations qu'entretiennent les veuves parisiennes de la fin du Moyen Âge avec leurs frères et sœurs couvrent ainsi un large éventail, allant de l'entraide fraternelle aux conflits les plus âpres : c'est là une des caractéristiques générales du lien adelphique qui, de la fusion à la haine, se décline sous une multitude de figures ${ }^{45}$. Soulignons cependant, au terme de ce rapide parcours, les principaux aspects qui illustrent la 
spécificité de la place des veuves au sein de leur fratrie. On retiendra tout d'abord le lien particulier qui unit les veuves au frère de leur mari défunt: qu'elle soit harmonieuse ou conflictuelle, la relation qui se noue entre eux est d'une importance primordiale, et se distingue des autres attachements fraternels par sa fréquence et son intensité ${ }^{46}$. On remarquera ensuite qu'au sein de leur fratrie, les veuves revêtent différents rôles qui s'entrecroisent et se superposent : sœurs germaines et belles-sœurs, maillon entre deux fratries, elles sont aussi héritières, douairières, exécutrices testamentaires, chefs de foyer fiscal ou encore mères. Cette identité plurielle, qu'estompe le seul terme de "veuves ", éclaire à bien des égards les différentes facettes de leur place et rôle de sœurs. Elle doit nécessairement être prise en compte dans le rapport à la fratrie.

Enfin, cet aperçu des liens "horizontaux » qui unissent les veuves à leurs frères et sœurs appelle une autre enquête, complémentaire, portant sur les liens "verticaux » qui s'établissent entre les veuves et leurs enfants. Dans les deux cas, ce qui interroge est l'impression d'oscillation des veuves entre autonomie et dépendance par rapport aux membres de leur parenté.

\section{NOTES}

1..Veve, mod. veuve, adj., « qui n'a plus de mari » (par suite de décès) : définition extraite de F. GODEFROY, Dictionnaire de l'ancienne langue française et de tous ses dialectes du IX au XVe siècle, t. 10, Paris, 1902, p. 852.

2..F. OLIVIER-MARTIN, Histoire de la coutume de la prévôté et vicomté de Paris, vol. 1, Paris, 1922-1930, réed. Paris, 1972, p. 228.

3..C'est aux XIV e et XVe siècles qu'apparaît en français le vocabulaire de la belle-famille tel que nous le connaissons : beau-frère est attesté à partir de 1386, et belle-sceur au début du $\mathrm{XV}^{\mathrm{e}}$ siècle, pour désigner le frère ou la sœur par alliance. Ces deux mots effacent l'emploi de serorge, issu du latin sororius (mari de la sœur), et désignant indifféremment le beau-frère ou la belle-sœur. Voir l'article « beau » dans J. DUBOIS, H. MITTERRAND, A. DAUZAT dir., Dictionnaire étymologique et historique du français, Paris, 1998, p. 73.

4..Voir sur ce thème Y. FOEHR-JANSSENS, La Veuve en majesté, Genève, 2000, p. 29-30.

5..Ce mode de désignation n'apparaît jamais dans les rôles d'impôt du XV siècle édités par Jean Favier, J. FAVIER, Les Contribuables parisiens à la fin de la Guerre de cent ans. Les rôles d'impôts de 1421, 1423 et 1438, Genève, 1970.

6..En revanche, il est parfois fait mention de fils, de filles, de gendres ou d'un second mari.

7..Pour le $\mathrm{XV}^{\mathrm{e}}$ siècle, nous disposons dans le minutier central des notaires de Paris des actes de sept études, dont deux seulement présentent une certaine unité et constituent une suite chronologique exploitable : les études VII et XIX (désormais abrégée en AN, MC ET XIX). Pour l'étude XIX, se référer à C. BÉCHU, F. GREFFE, I. PEBAY, Minutier central des notaires de Paris, minutes du XVe siècle de l'étude XIX, inventaire analytique, Paris, 1993.

8..Nous nous appuierons sur le registre des causes civiles du Châtelet de Paris.

9..En mars 1319-1320, l'article 17 de l'ordonnance royale concernant les baillis et les sénéchaux rappelle ce nécessaire soutien des veuves : «Que les femmes veuves et les orphelins il garderont, et s'il ont à faire 
pardevant eulx, soit en assise ou ailleurs, il leur avanceront leur droit, et les delivreront tous premiers avant tous autres en la meilleur maniere qu'il pourront. ", Ordonnances des roys de France de la troisième race, vol. XII, Paris, 1723-1849, p. 450.

10..Cette protection consiste en une assistance morale, mais aussi juridique, du moins jusqu'au début du XIV ${ }^{e}$ siècle, époque à laquelle l'Église perd sa juridiction sur les veuves. Jusqu'alors, le droit canonique soustrayait sans difficulté les veuves à la juridiction ordinaire pour leur assurer le privilège juridictionnel des cours ecclésiastiques. Sur ce point, voir R. METZ, La Femme et l'Enfant dans le droit canonique médiéval, Londres, 1985 (éd. or. 1951-1976), p. 91 sq.

11..Codicille d'Arnaud de Corbie du 9 août 1407, Paris, BnF, collection Moreau, 1161, fo $744 \mathrm{v}^{\circ}$, cité par A. TUETEY, Testaments enregistrés au Parlement de Paris sous le règne de Charles VI, Paris, 1880 (Collections de documents inédits sur l'Histoire de France, Mélanges historiques, t. III), p. 285.

12..Testament de Renaud de Trie du 12 mai 1406, Paris, AN, $X^{1 A} 9807$, fo $185 \mathrm{v}^{\circ}$, cité par A. TUETEY, op. cit., p. 417.

13..Paris, AN, MC ET XIX/7, $n^{\circ} 1607,21$ août 1492.

14..Paris, AN MC ET XIX/12, n 3745, 11 septembre 1497.

15..S'adressant aux veuves dans le Livre des trois vertus (troisième partie, chapitre IV), Christine de Pizan écrit : « Ou fait des plaiz qui vous assauldront (...) devez savoir que eschiver devez plait et procés tout le plus que vous pouez ; car c'est chose qui trop puet grever femme veuve pour pluseurs raisons : l'une que elle ne s'i cognoist et est simple en telz choses ; l'autre que il convient que elle se mette en dongier d'autrui pour faire solliciter ses besoignes, et gens sont communement mal diligens des besoingnes aux femmes et voulentiers les trompent et mettent en despens ·VIII. sols pour ·VI· ; et l'autre qu'elle n'y puet a toutes heures aller comme feroit un home, et pour ce est le meilleur conseil que elle laisse avant aller aucune partie de son droit, mais que ce ne soit a trop grant oultraige que elle s'i fiche. », CHRISTINE DE PIZAN, Le Livre des trois vertus, C.C. WILLARD éd., Paris, 1989, p. 190.

16..CHRISTINE DE PIZAN, Le Livre des fais et bonnes meurs du sage roy Charles V, S. SOLENTE éd., Paris, 1936, p. XVI.

17..F. OLIVIER-MARTIN, Histoire de la coutume... op. cit., vol. 1, Paris, 1922-1930, réed. Paris, 1972, p. 204 sq.

18..C'est également ce qu'observe Emmanuelle Santinelli en ce qui concerne le haut Moyen Âge : la protection personnelle des veuves apparaît en premier lieu comme le devoir de leurs parents mâles, E. SANTINELLI, Des Femmes éplorées? Les veuves dans la société aristocratique du haut Moyen Âge, Villeneuve d'Ascq, 2003, p. 62-63.

19. Sur le phénomène de « parentification » des frères et sœurs aînés au Moyen Âge, voir D. LETT, « La sorella maggiore «madre sostitutiva» nei miracoli di san Luigi », dans A. ARRU, S. BOESCH GAJANO dir., Fratello e sorella, Quaderni Storici, 2, 1993, p. 341-353; et ID., « Vieux frères et oncles jeunes : écart de générations et écart d'âge dans les familles de la fin du Moyen Âge », dans J.-P. BARDET, J.-N. LUC, I. ROBINROMERO et C. ROLLET dir., Lorsque l'enfant grandit. Entre dépendance et autonomie, Paris, 2003, p. 97.

20..Paris, AN, MC ET XIX/1, n 34, 30 mai 1483 et XIX/6 , n 1198, 13 septembre 1491.

21..Paris, AN, MC ET XIX/4, $\mathrm{n}^{\circ} 700,10$ mai 1489.

22. Testament de Simonnette la Maugère du 26 octobre 1420, Paris, BnF, collection Moreau, 1162, fo $428 \mathrm{r}^{\circ}$, cité par A. TUETEY, op. cit., p. 622.

23..Paris, AN, MC ET XIX/13, nº 4194, 27 mars 1499.

24..Paris, AN, MC ET XIX/4, $n^{\circ} 756,12$ septembre 1489.

25..Paris, AN, MC ET XIX/13, nº 4150, 4 novembre 1498.

26..Paris, AN, MC ET XIX/11, $n^{\circ} 2918,6$ avril 1496.

27..Paris, AN, MC ET XIX/16, n 5117, 24 novembre 1500. 
28..Peut-être faut-il voir ici un processus de « masculinisation » de la veuve, tel qu'il est observé par certains anthropologues et historiens. Voir les exemples de veuves viriles fournis par G. BALANDIER, Anthropologiques, Paris, 1974, p. 38 sq., F. HÉRITIER, Masculin/Féminin. La pensée de la différence, Paris, 1996, p. 246-247, et K. MEYER-ROUX, «Quella virile vedova : la prophétesse Anne comme veuve modèle dans la Toscane des XIVe et $\mathrm{XV}^{\mathrm{e}}$ siècles ", dans I. CHABOT, J. HAYEZ et D. LETT dir., La famille, les femmes et le quotidien (XIV ${ }^{e}-\mathrm{XVIII}{ }^{e}$ siècle). Textes offerts à Christiane Klapisch-Zuber, Paris, 2006, p. 215-237.

29..Voir sur ce point F. OLIVIER-MARTIN, Histoire de la coutume..., op. cit., p. 241-242.

30..Paris, AN, MC ET XIX/10, n² 2582, 24 juillet 1495 ; Paris, AN, MC ET XIX/12, $n^{\circ} 4082,1^{\text {er }}$ avril 1498.

31..On pense ici au mariage léviratique observé par les anthropologues. Dans le cadre du lévirat, le frère peut apparaître comme un « substitut » du mari défunt, M. AUGÉ, Les Domaines de la parenté. Filiation, alliance, résidence, Paris, 1975, p. 34-35. Par ailleurs, le lévirat permet de maintenir les relations d'alliance matrimoniale entre les clans, P. BONNEMÈRE, Le Pandanus rouge. Corps, différence des sexes et parenté chez les Ankave-Anga (Papouasie-Nouvelle-Guinée), Paris, 1996, p. 80.

32..J. PETIT, Registre des causes civiles de l'officialité épiscopale de Paris (1384-1387), Paris, 1919.

33..Pour davantage de précisions, voir J.-PH. LEVY, «L'officialité de Paris et les questions familiales à la fin du XIVe siècle », dans Études d'histoire du droit canonique dédiées à Gabriel Le Bras, vol. 2, Paris, 1965, p. 1265-1294.

34..D'après nos relevés, quand les enfants sont orphelins de père, sur 23 cas de dation de tuteurs et curateurs recensés dans le registre, la mère des enfants, veuve, est nommée 19 fois en tant que membre de ce conseil de famille.

35..Voir sur ce point D. LETT, « Vieux frères et oncles jeunes... », loc. cit., p. 100.

36..F. OLIVIER-MARTIN, Histoire de la coutume..., op. cit., n 189, février 1505.

37..Accordé aux veuves « qui demeurent esbahies et desconfortées » à la mort de leur mari (PHILIPPE DE BEAUMANOIR, Coutumes de Beauvaisis, Paris, 1899-1900, n 429), le douaire consiste plus précisément en un droit de jouissance viagère portant sur une partie des propres du mari. Il s'agit d'une dotation faite par le mari à son épouse au moment du mariage. Géré en usufruit pendant l'union conjugale, il doit servir à faire vivre la femme si son mari décède avant elle.

38..Une femme mariée ne peut être assignée en justice sans que son mari le soit en même temps pour l'autoriser. Voir sur ce point F. OLIVIER-MARTIN, Histoire de la coutume... op. cit., vol. 1, Paris, 1922-1930, réed. Paris, 1972, p. 222 ; et JACQUES D’ABLEIGES, Le Grand Coutumier de France, Paris, 1868, p. 323 : « Se une femme vefve est en procès et elle se marie, il convient faire adjourner son mary à reprendre les erremens ».

39..F. OLIVIER-MARTIN, Sentences civiles du Châtelet de Paris, publiées d'après les registres originaux, Paris, 1914 $\mathrm{n}^{\circ}$ 207, 20 février 1399.

40..G. FAGNIEZ, loc. cit., $\mathrm{n}^{\circ}$ 71, 27 février 1399.

41..Le testateur désigne en général plusieurs exécuteurs testamentaires, choisis parmi un cercle de proches parents et d'amis. Très souvent, la veuve est désignée comme exécutrice testamentaire.

42..G. FAGNIEZ, loc. cit., $\mathrm{n}^{\circ} 71,27$ février 1399 : « ledit frere et heritier disoit que de raison et par la generale coustume du royaume de France par laquele le mort saisist le vif, son hoir, il estoit saisy de tous les biens, meubles et immeubles demourés du decès dudit defunct son frere ». Voir sur ce point F. OLIVIER-MARTIN, Histoire de la coutume... op. cit., vol. 2, Paris, 1922-1930, réed. Paris, 1972, p. 431 et p. 533-534.

43.J. PETIT, op. cit., col. 499 : Guillaume des Lions contre Agnès.

44.Voir sur le remariage des veuves les analyses de CH. KLAPISCH-ZUBER : « Par le remariage de la veuve de leur sang, les Florentins affirment qu'ils n'ont jamais renoncé totalement à contrôler les dots qu'ils ont données à leurs filles ou à leurs sœurs ", et EAD., «La "mère cruelle"». Maternité, veuvage et dot dans la Florence des XIVe-XVe siècles ", Annales ESC., 5, 1983, p. 1100.

45..D. LETT, Histoire des frères et sœurs, Paris, 2004. 
46..C'est également ce que souligne I. RÉAL lorsqu'elle étudie la fraternité par alliance dans les sources hagiographiques : « Le cas de figure le plus fréquent montre en fait les relations entre le frère du mari et sa belle-sœur veuve. Son attitude à son égard se veut en théorie fraternelle, autrement dit fondée sur le devoir de protection. ", Vies de saints, vie de famille. Représentation et système de la parenté dans le royaume mérovingien (481-751) d'après les sources hagiographiques, Turnhout, 2001, p. 491.

\section{RÉSUMÉS}

À la fin du Moyen Âge, à Paris, les veuves entretiennent avec leurs fratries des relations diverses. Les legs testamentaires ou les actes notariés montrent que les veuves, surtout dans les classes aisées, bénéficient de façon privilégiée d'une protection financière ou d'une entraide fraternelle. Cette assistance est principalement masculine. En même temps, les veuves ne sont pas évincées, du fait de la mort de leur mari, des affaires et décisions familiales : pleinement concernées, elles y participent de façon active. Le registre des causes civiles du Châtelet de Paris contient nombre d'affaires dans lesquelles les veuves s'opposent à leurs frères et sœurs devant les tribunaux. Les conflits surviennent surtout quand les veuves souhaitent entrer en possession de leur héritage, et particulièrement de leur douaire, ou décident de se remarier.

Parisian widows and their sibship. At the end of the Middle Ages, in Paris, the ties between widows and their siblings are various. Wills and deeds show that the widows, especially in the leading classes of society, benefit from financial aid and support from their siblings. This is mainly a masculine assistance. Nevertheless, widows are not ousted from family affairs and decisions: they are fully concerned and play an active part. We find in the records of the Chatelet court in Paris examples of trials in which widows were involved against their brothers or sisters. Conflicts often occur when widows want to come into their inheritance, particularly their dower, or decide to remarry.

\section{INDEX}

Mots-clés : droit, familles, femmes, veuves

Keywords : family, law, Paris, widows, women

\section{AUTEUR}

\section{CAROLINE JEANNE}

Université Paris I-Panthéon-Sorbonne - LAMOP, UMR 8589, 17 rue de la Sorbonne, 75005 Paris 